\title{
Adamantinoma-Like Ewing Sarcoma of the Thyroid: A Case Report and Review of the Literature
}

\author{
Diana Morlote ${ }^{1} \cdot$ Shuko Harada $^{1} \cdot$ Brenessa Lindeman $^{2} \cdot$ Todd M. Stevens $^{1}(\mathbb{C}$
}

Received: 6 December 2018 / Accepted: 3 February 2019 / Published online: 8 February 2019

(c) Springer Science+Business Media, LLC, part of Springer Nature 2019

\begin{abstract}
Currently considered a variant of Ewing sarcoma, adamantinoma-like Ewing sarcoma is a rare malignancy that shows classic Ewing sarcoma-associated gene fusions but also epithelial differentiation. Here we present the 6th reported case of adamantinoma-like Ewing sarcoma involving the thyroid gland. Sections of the thyroid tumor from a 20 -year old woman showed sheets, lobules and trabeculae of primitive, uniform, small round blue cells that diffusely expressed pankeratin, $\mathrm{p} 40$ and CD99. Fluorescent in situ hybridization revealed an EWSRI gene rearrangement and an EWSRI-FLII fusion was detected by RT-PCR. Neck lymph nodes were not involved, and the patient was treated with a Ewing sarcoma chemotherapy protocol and radiation and is disease free 7 months after surgery. The unusual histology and immunohistochemical profile of adamantinoma-like Ewing sarcoma makes diagnosis and classification very challenging. We also present a literature review of adamantinoma-like Ewing sarcoma involving the thyroid.
\end{abstract}

Keywords Adamantinoma-like Ewing sarcoma $\cdot$ Thyroid $\cdot$ Sarcoma $\cdot$ Ewing sarcoma $\cdot$ Molecular diagnostics

\section{Introduction}

Adamantinoma-like Ewing sarcoma (ALES), a rare member of the Ewing family of tumors, was originally described in the long bones; however, reports of cases in the thyroid, sinonasal tract, orbit, and salivary gland have surfaced during the last 10 years [1-5]. In fact, it appears the head and neck is the site most commonly affected by ALES [5].

Histologically, ALES grows in sheets, lobules, nests and trabeculae composed of monotonous, uniform round cells with evenly distributed and vesicular nuclear chromatin similar to Ewing sarcoma. Cytoplasm is scant to clear, psuedorosettes may be present, and mitotic activity is abundant. Like classic Ewing sarcoma (ES), membranous CD99 expression is typical. Unlike classic Ewing sarcoma, however, ALES features complex epithelial differentiation in the form of variable cohesive growth, squamous pearls,

Todd M. Stevens

tstevens@uabmc.edu

1 Department of Pathology, University of Alabama at Birmingham, 3548 North Pavilion, 1802 6th Ave. S, Birmingham, AL 35249, USA

2 Department of Surgery, University of Alabama at Birmingham, Birmingham, AL, USA intercellular bridges, and peripheral palisading, and they diffusely express epithelial markers such as p40 and keratins. By definition, ALES carry EWSRl gene rearrangements. So far, the partner gene has consistently been FLII [1, 4]. Their rarity and unusual histologic and immunohistochemical profile present a diagnostic challenge. Here we present a case of ALES involving the thyroid and review the literature on intrathyroidal ALES.

\section{Case Report}

A 20-year-old woman presented with a non-painful growth on the left neck recently noticed by her mother. An ultrasound found a $4 \mathrm{~cm}$ solid nodule with punctate calcifications within the left lobe of the thyroid. Fine needle aspiration was interpreted as suspicious for carcinoma. A ThyroSeq panel revealed loss of PTEN; however, this assay does not distinguish between germline and somatic mutations. A total thyroidectomy with left central neck dissection was performed. The thyroid tumor histologically featured a primitive small round blue cell tumor growing in sheets, nests, lobules, cords and trabeculae (Fig. 1a-d). There was colonization of the background thyroid follicles by the tumor cells (Fig. 1b), which were set in a finely fibrous/hyalinized 

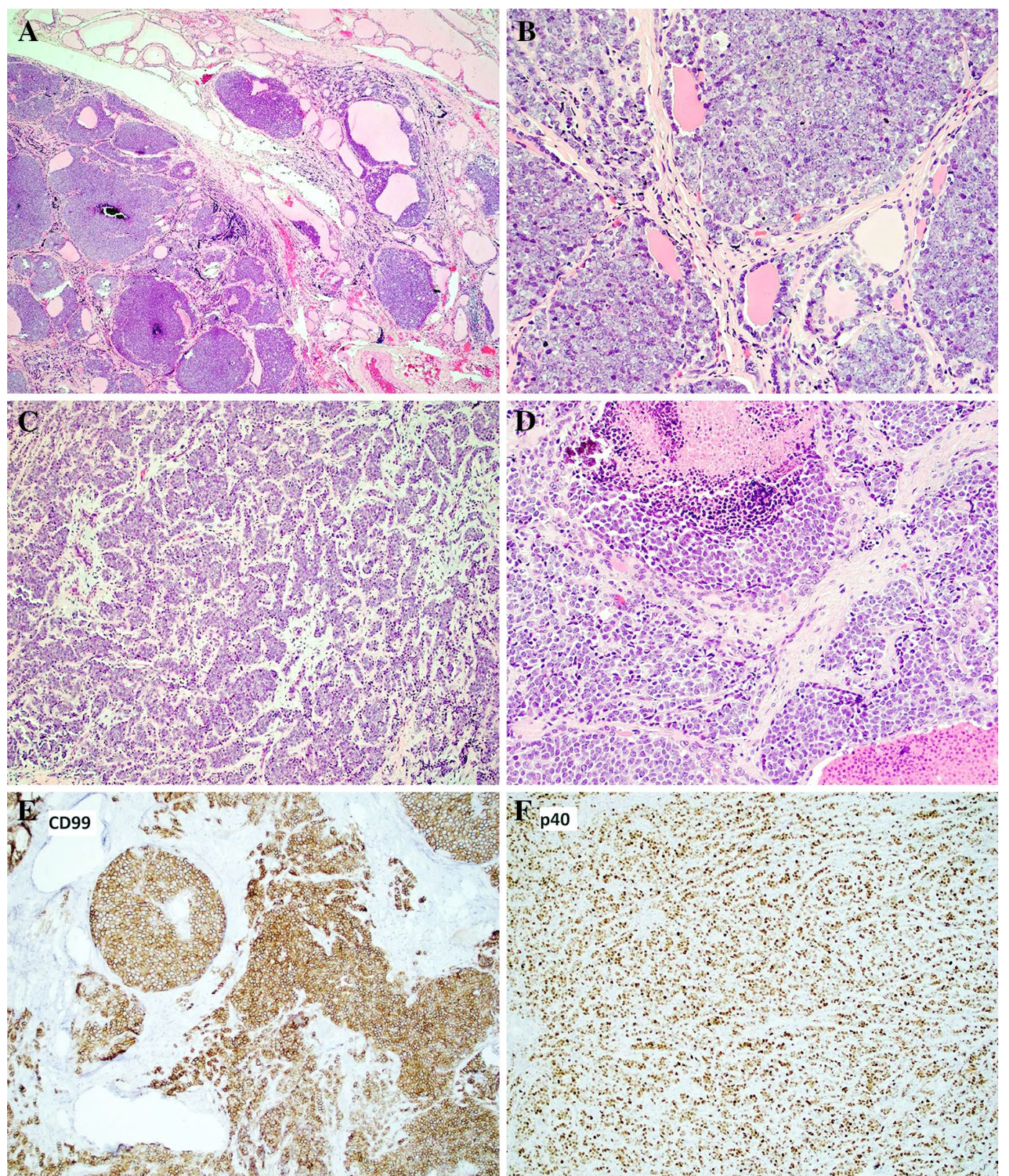

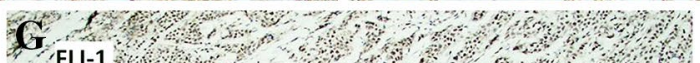

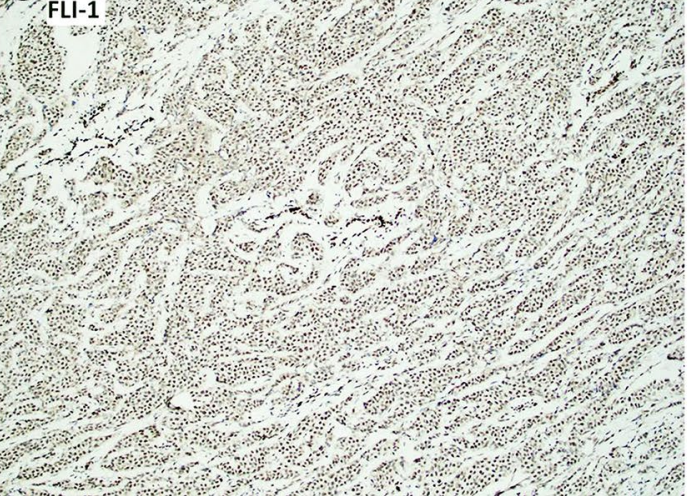


4Fig. 1 Adamantinoma-like Ewing Sarcoma arising in the thyroid of a 20 year-old female. Sheets, islands and trabeculae of tumor cells (a, b, c, d, 100× $400 \times, 200 \times, 400 \times$, respectively, hematoxylin and eosin) showing colonization of thyroid follicles (b) and necrosis (d). Tumor cells were set in a fibrous/hyalinized and myxoid stroma (c, d). Cells were uniform, small to medium in size, with round nuclei, fine chromatin and variably prominent nucleoli (b). Immunohistochemical stains revealed strong, membranous expression of CD99 (e, $400 \times)$ and diffuse nuclear expression of p40 (f, 200×) and FLI-1 (g, 200×). RT-PCR revealed a EWSR1-FLI-1 fusion. Immunostains for thyroglobulin, TTF1, PAX8, Calcitonin, CK20, SOX10, ERG, SMA, and NUT were all negative (not shown)

and myxoid stroma (Fig. 1c, d). The neoplastic cells were uniform, small to medium in size, with round nuclei, evenly distributed chromatin and a variably prominent nucleolus. Cytoplasm was scant to eosinophilic (Fig. 1d). Occasional somewhat plasmacytoid cells were noted. There were suggestions of pseudorosette formation as well as scattered calcifications. Mitotic rate was $10-20$ mitoses/10 hpf and there was abundant apoptotic debris and multifocal necrosis (Fig. 1d). Overall, the cytologic appearance was more typical of Ewing sarcoma than carcinoma. No squamous pearls or intercellular bridges were seen. However, immunohistochemical stains showed evidence of epithelial differentiation (diffusely positive for Pankeratin and p40) (Fig. 1f). Tumor cells were also positive for CD99 (diffuse, strong and membranous) (Fig. 1e) and FLI1 (nuclear) (Fig. 1g), while rare cells were positive for synaptophysin. The following stains were negative: Thyroglobulin, TTF1, PAX8, Calcitonin, CK20, SOX10, ERG, SMA, and NUT. Fluorescent in-situ hybridization revealed an EWSRI gene rearrangement and a EWSRI-FLII fusion was detected by RT-PCR. Given the morphological, immunohistochemical and molecular findings, a diagnosis of Ewing sarcoma with epithelial differentiation (adamantinoma-like Ewing sarcoma) was rendered. Ten lymph nodes were negative for malignancy. No metastatic disease was detected in this case. The patient is receiving a Ewing sarcoma chemotherapy regimen (14 cycles of vincristine, doxorubicin, cyclophosphamide alternating with ifosfamide, etoposide) and radiation for a focally positive margin (5040 cGy in 28 fractions to the left thyroidectomy bed) and remains disease-free by imaging 7 months after diagnosis.

\section{Discussion}

While not exactly histologically reminiscent of adamantinoma of bone, ALES is so named because it was originally reported in long bone sites, where a major differential diagnostic consideration was adamantinoma. As currently defined, ALES is a small round blue cell tumor harboring the EWSRI-FLII fusion along with epithelial differentiation at the light microscopic and/or immunohistochemical level $[1,5]$.

Cruz et al. [6] and Eloy et al. [7, 8] initially reported two thyroid small round blue cell tumors that showed diffuse expression of p63, pankeratin and CD99 and harbored EWSRI-FLII fusions under the name "carcinoma of the thyroid with Ewing family tumor elements." Both of these cases showed essentially a small round blue cell tumor with scant cytoplasm growing in solid and insular patterns with epidermoid foci noted in one case that was initially diagnosed as a small cell carcinoma [6]. Subsequently, Bishop et al. [1] reported two additional thyroid tumors featuring nested, basaloid and trabecular growth with interlobular fibrosis, variable peripheral palisading and focal rosette formation, composed of small, uniform cells with minimal cytoplasm; one example showed a microcystic pattern set in a myxoid stroma. Both of these cases expressed pankeratin, p40, CD99 and harbored EWSRI-FLII fusions. Because EWSRI-FLII fusions are currently pathognomonic for Ewing sarcoma, Bishop et al. [1] preferred the terminology adamantinomalike Ewing sarcoma for these cases. Like the new case reported herein, one of the cases from Bishop et al. [1] showed colonization of the thyroid follicles.

The two cases reported by Cruz et al. [6], Eloy et al. [7], and Eloy and Oliveira et al. [8] had good outcomes, with the patients showing no evidence of disease at 38 months and 13 years, respectively. Ongkeko et al. [9] reported a thyroid ALES that histologically showed a basaloid malignancy with focal peripheral palisading composed of nests of monotonous round cells with scant cytoplasm with areas of follicular colonization. The Ongkeko et al. case featured pancreatic and neck node metastasis, yet a restaging PET scan showed complete response 2 years after chemotherapy. That the case reported by Ongkeko was initially diagnosed as poorly differentiated thyroid carcinoma underscores the challenge in accurately diagnosing ALES. The available clinical, treatment, outcome and immunohistochemical features of the now six cases of ALES to involve the thyroid are presented in Tables 1 and 2. The age range of affected patients is 19-42 (mean: 29.5).

There is debate regarding if ALES is a variant of ES, or is rather a type of carcinoma harboring a EWSRI-FLI rearrangement. Arguments for the former are that EWSRI-FLII fusions at this time are considered pathognomonic for ES [5], ES has been shown to express keratins and may show atypical histologic patterns such as large cell, spindle cell, and sclerosing patterns [10], and cases of ALES will still show areas with morphologic similarity to classic ES. Features favoring the latter argument are that many examples of tumors exist that are clearly different yet harbor the same or similar gene rearrangements (for example, clear cell sarcoma of soft parts and hyalinizing clear cell carcinoma, etc.) [11]. 
Table 1 Summary of the salient therapeutic and outcome data of the reported cases of adamantinoma-like Ewing sarcoma of the thyroid

\begin{tabular}{|c|c|c|c|c|c|c|}
\hline Reference & Age/sex & Fusion & Surgery/adjuvant treatment & Follow up/outcome & LN metastasis & Distant metastasis \\
\hline $\begin{array}{l}\text { Cruz et al. and Eloy } \\
\text { et al.^ }[6,7]\end{array}$ & $42 / \mathrm{F}$ & EWSR1-FLII & TT/none & 38 months/ANED & $\mathrm{N}$ & $\mathrm{N}$ \\
\hline Eloy et al.^ $[8]$ & 24/M & EWSR1-FLI1 & TT/RAI/ESP & 13 years/ANED & $\mathrm{N}$ & $\mathrm{N}$ \\
\hline Bishop et al. [1] & 19/M & EWSR1-FLII & TT/unknown & $\mathrm{n} / \mathrm{a}$ & $\mathrm{n} / \mathrm{a}$ & $\mathrm{n} / \mathrm{a}$ \\
\hline Bishop et al. [1] & $36 / \mathrm{F}$ & EWSR1-FLII & TT/unknown & $\mathrm{n} / \mathrm{a}$ & $\mathrm{n} / \mathrm{a}$ & $\mathrm{n} / \mathrm{a}$ \\
\hline Ongkeko et al. [9] & $36 / \mathrm{M}$ & EWSR1-FLII & $\begin{array}{l}\text { TT/Radiation after initial diag- } \\
\text { nosis of PDTC; chemotherapy }\end{array}$ & $\begin{array}{l}2 \text { years/alive, restag- } \\
\text { ing PET showed } \\
\text { complete response }\end{array}$ & $\mathrm{Y}$ & $\mathrm{Y}$ (pancreas) \\
\hline Current case & $20 / \mathrm{F}$ & EWSR1-FLI1 & TT/ESP/radiation & $\begin{array}{l}7 \text { months/ANED; } \\
\text { PET scan NED at } 7 \\
\text { months }\end{array}$ & $\mathrm{N}$ & $\mathrm{N}$ \\
\hline
\end{tabular}

n/a not available, $N$ no, $Y$ yes, $T T$ total thyroidectomy, $R A I$ radioactive iodine, ESP Ewing sarcoma therapy protocol, $A N E D$ alive no evidence of disease, $P D T C$ poorly differentiated thyroid carcinoma

$\wedge$ Reported under the name "carcinoma of the thyroid with Ewing family tumor elements"

Table 2 Summary of the immunohistochemical features of the six reported cases of adamantinoma-like Ewing sarcoma of thyroid

\begin{tabular}{|c|c|c|c|c|c|c|c|c|c|c|c|c|c|c|c|c|}
\hline Reference & age/sex & CD99 & p63 & $\mathrm{p} 40$ & Pan-CK & SYN & CHR & TTF1 & TG & Calcitonin & PAX8 & S100 & SOX10 & Desmin & SMA & NUT-1 \\
\hline $\begin{array}{l}\text { Cruz et al. and } \\
\text { Eloy et al.^ } \\
{[6,7]}\end{array}$ & $42 / F$ & + & + & $\mathrm{n} / \mathrm{a}$ & + & - & - & - & - & - & $\mathrm{n} / \mathrm{a}$ & - & $\mathrm{n} / \mathrm{a}$ & $\mathrm{n} / \mathrm{a}$ & - & $\mathrm{n} / \mathrm{a}$ \\
\hline Eloy et al.^ [8] & $24 / \mathrm{M}$ & + & + & $\mathrm{n} / \mathrm{a}$ & + & - & - & - & - & - & $\mathrm{n} / \mathrm{a}$ & - & $\mathrm{n} / \mathrm{a}$ & $\mathrm{n} / \mathrm{a}$ & $\mathrm{n} / \mathrm{a}$ & $\mathrm{n} / \mathrm{a}$ \\
\hline Bishop et al. [1] & $19 / \mathrm{M}$ & + & $\mathrm{n} / \mathrm{a}$ & $f+$ & + & - & - & $\mathrm{n} / \mathrm{a}$ & $\mathrm{n} / \mathrm{a}$ & $\mathrm{n} / \mathrm{a}$ & $\mathrm{n} / \mathrm{a}$ & $\mathrm{f}+$ & $\mathrm{n} / \mathrm{a}$ & - & $f+$ & - \\
\hline Bishop et al. [1] & $36 / F$ & + & $\mathrm{n} / \mathrm{a}$ & + & + & + & $f+$ & $\mathrm{n} / \mathrm{a}$ & $\mathrm{n} / \mathrm{a}$ & $\mathrm{n} / \mathrm{a}$ & $\mathrm{n} / \mathrm{a}$ & - & $\mathrm{n} / \mathrm{a}$ & - & - & $\mathrm{n} / \mathrm{a}$ \\
\hline Ongkeko et al. [9] & $36 / \mathrm{M}$ & + & $\mathrm{n} / \mathrm{a}$ & $\mathrm{n} / \mathrm{a}$ & + & - & - & - & - & - & $\mathrm{n} / \mathrm{a}$ & - & $\mathrm{n} / \mathrm{a}$ & $\mathrm{n} / \mathrm{a}$ & $\mathrm{n} / \mathrm{a}$ & $\mathrm{n} / \mathrm{a}$ \\
\hline Current case & $20 / \mathrm{F}$ & + & $\mathrm{n} / \mathrm{a}$ & + & + & + & $\mathrm{n} / \mathrm{a}$ & - & - & - & - & $\mathrm{n} / \mathrm{a}$ & - & $\mathrm{n} / \mathrm{a}$ & - & - \\
\hline
\end{tabular}

$f$ focal, Pan-CK Pan-cytokeratin, SMA smooth muscle actin, SYN synaptophysin, $C H R$ chromogranin, $T G$ thyroglobulin, $n / a$ not available

${ }^{\wedge}$ Reported under the name "carcinoma of the thyroid with Ewing family tumor elements"

Future studies and experience with ALES may answer this classification and etiologic question.

The differential diagnosis of ALES involving the thyroid is with poorly differentiated (insular) thyroid carcinoma (PDTC), small cell variant of medullary carcinoma, spindle epithelial tumor with thymus-like differentiation (SETTLE), and carcinoma showing thymus-like elements (CASLTE)/ intrathyroid thymic carcinoma $[1,9]$. PDTC, while showing architectural overlap with ALES, may feature areas of a more well-differentiated thyroid carcinoma and should retain expression of TTF1 and thyroglobulin and will lack EWSRI-FLII rearrangements. Medullary carcinoma can be excluded due to the absence of amyloid and lack of calcitonin and TTF1 expression by ALES [12]. SETTLE is a rare entity of presumed branchial origin which also shows diffuse expression of keratins but also features spindled, glomeruloid, and possibly mucocyte differentiation, will lack the EWSR1-FLII fusion, and will show less nuclear atypia and lower mitotic count than ALES [13]. Intrathyroid thymic carcinoma, like ALES, will show expression of epithelial markers but it typically is more squamous in appearance with variable lymphoid infiltrate and may also express CD5 and CD117 [9].

When presenting in the parotid or submandibular gland, ALES typically shows less overt evidence of keratinization and may also feature basement membrane deposition [5] and thus may easily be misdiagnosed as basal cell adenoma, basal cell adenocarcinoma, and high-grade adenoid cystic carcinoma [5]. Basal cell adenoma and adenocarcinoma, however, will typically show low grade cytology, much lower mitotic count, and will typically feature a biphasic luminal and myoepithelial pattern of staining with p40 or p63, and will be negative for CD99, unlike ALES. Highgrade adenoid cystic carcinoma (ACC) will often, in addition to the transformed component, show areas of classic ACC patterns such as lower-grade, angulated cells forming tubular and/or cribriform structures with spaces containing basophilic material that will also feature a dual cell population on $\mathrm{p} 63$ or $\mathrm{p} 40$ staining, whereas ALES will show all or most cells staining for $\mathrm{p} 40$ or p63. Molecular study will help 
resolve difficult cases as ACC contain $M Y B$ or $M Y B L 1$ rearrangements and lacks EWSRl fusions [14]. Merkel cell carcinoma could pose a diagnostic challenge with ALES given that it also expresses neuroendocrine markers and features similar cytologic features [15]. However, Merkel cell carcinoma will lack $E W S R I$ rearrangements and show perinuclear dot-like reactivity for CK20. Given rosette formation and variable synaptophysin expression in ALES, a high-grade neuroendocrine carcinoma is also in the differential diagnosis [5]. However, neuroendocrine carcinoma will typically lack the extensive p40 or p63 expression seen in ALES and will typically show more cytologic variability. Basaloid squamous cell carcinoma (BSCC) should also be excluded in any head and neck site before diagnosing ALES. BSCC will overlap immunophenotypically with ALES although CD99 expression should be absent in BSCC. In addition, BSCC will show more nuclear pleomorphism and may be associated with an in situ surface component and lack EWSRI gene rearrangements [16].

In the sinonasal tract, sinonasal undifferentiated carcinoma (SNUC), HPV-related multiphenotypic carcinoma, and olfactory neuroblastoma are mimics of ALES. SNUC, like ALES, may show limited neuroendocrine marker expression, however, SNUC, by definition, lacks squamous differentiation and thus should be mostly negative for squamous markers such as p40, unlike ALES. HPV-related multiphenotypic carcinoma (HMSC) is a neoplasm that is so far restricted to the sinonasal tract and, like ALES, will also feature basaloid cells with high mitotic rates and necrosis. However, unlike ALES, surface dysplasia will typically be present and HMSC will typically feature cribriform and ductal areas with a variable two-cell population of inner luminal cells surrounded by outer myoepithelial cells [14]. Of course, HMSC will be positive for high-risk HPV, typically type 33, and will lack EWSRI rearrangement. Olfactory neuroblastoma will typically involve the cribriform plate and may feature neuropil and an S100-positive sustentacular network, and is usually negative for keratins, unlike ALES [17].

NUT carcinoma, myoepithelial carcinoma, synovial sarcoma, and lymphoma are additional differential diagnosis to consider before diagnosing ALES. NUT carcinomas can overlap with ALES at the architectural, cytologic and immunophenotypic levels but the highly specific and sensitive NUT monoclonal antibody will be negative in ALES [18]. Myoepithelial carcinomas can feature small round blue cell histology that often express p40 and keratins and may contain EWSR 1 rearrangements in a significant subset, thus showing a potential for remarkable overlap with ALES. However, unlike ALES, myoepitheial carcinomas lack the EWRS1-FLI1 rearrangement and instead show EWSRI fused to either POU5F1, PBX1, PBX3, ZNF44, or KLF15 [11]. A biphasic synovial sarcoma would not be difficult to separate from ALES. However, in a small biopsy specimen, an
ALES could be confused for monophasic synovial sarcoma, especially since synovial sarcoma can express CD99 [9]. Synovial sarcoma, however, will typically show only focal expression of keratins unlike the diffuse expression typical of ALES, and will also harbor SS18 gene rearrangements. $\mathrm{B}$ and $\mathrm{T}$ acute lymphoblastic lymphoma/leukemia express CD99 and could enter the differential diagnosis of ALES on a small biopsy specimen but can be easily excluded with a basic immunohistochemical panel including TdT [19].

Accurate diagnosis of ALES is crucial for establishing prognosis and guiding therapy, as so far ALES have been typically treated with ES- specific chemotherapy regimens [5]. This case and others $[1,5,9]$ highlights that overt epithelial differentiation in a thyroid tumor, or for that matter a tumor from any site, does not by itself exclude an Ewing family tumor. Poorly differentiated head and neck tumors that show nuclear monotony, fine chromatin, epithelial differentiation and positivity for CD99 should prompt further immunohistochemical and possibly molecular workup to exclude ALES. Molecular confirmation is necessary to make a definitive diagnosis of ALES. So far, all cases of ALES both within and outside the thyroid have shown an EWSRIFLII fusion $[1,9,20]$. While too few cases of ALES of thyroid have been reported to make a definitive statement on its prognosis, it appears ALES of thyroid (and other head and neck sites) appears to carry a more favorable prognosis as compared to classic ES. Thus, awareness of this tumor type will help facilitate its recognition and drive further studies that may shed further insights into this rare cancer.

\section{Compliance with Ethical Standards}

Conflict of interest The authors have no conflicts of interest or financial relationships to disclose.

Ethical Approval All procedures performed in studies involving human participants were in accordance with the ethical standards of the institutional and/or national research committee and with the 1964 Helsinki declaration and its later amendments or comparable ethical standards.

Ethical Approval This article does not contain any studies with animals performed by any of the authors.

Informed Consent Informed consent was waived for this study as part of the corresponding author's IRB at UAB (X170428002).

\section{References}

1. Bishop JA, Alaggio R, Zhang L, Seethala RR, Antonescu CR. Adamantinoma-like Ewing family tumors of the head and neck: a pitfall in the differential diagnosis of basaloid and myoepithelial carcinomas. Am J Surg Pathol. 2015;39:1267.

2. Weinreb I, Goldstein D, Perez-Ordoñez B. Primary extraskeletal Ewing family tumor with complex epithelial differentiation: a 
unique case arising in the lateral neck presenting with Horner syndrome. Am J Surg Pathol. 2008;32:1742-8.

3. Kikuchi Y, Kishimoto T, Ota S, et al. Adamantinoma-like Ewing family tumor of soft tissue associated with the vagus nerve: a case report and review of the literature. Am J Surg Pathol. 2013;37:772-9.

4. Lezcano C, Clarke MR, Zhang L, Antonescu CR, Seethala RR. Adamantinoma-like Ewing sarcoma mimicking basal cell adenocarcinoma of the parotid gland: a case report and review of the literature. Head Neck Pathol. 2015;9:280-5.

5. Rooper LM, Jo VY, Antonescu CR, et al. Adamantinoma-like Ewing sarcoma of the salivary glands: a newly recognized mimicker of basaloid salivary carcinomas. Am J Surg Pathol. 2019;43:187-94.

6. Cruz J, Eloy C, Aragüés JM, Vinagre J, Sobrinho-Simões M. Small-cell (basaloid) thyroid carcinoma: a neoplasm with a solid cell nest histogenesis? Int J Surg Pathol. 2011;19:620-6.

7. Eloy C, Cameselle-Teijeiro J, Vieira J, et al. Carcinoma of the thyroid with Ewing/PNET family tumor elements: a tumor of unknown histogenesis. Int J Surg Pathol. 2014;22:579-81.

8. Eloy C, Oliveira M, Vieira J, et al. Carcinoma of the thyroid with Ewing family tumor elements and favorable prognosis: report of a second case. Int J Surg Pathol. 2014;22:260-5.

9. Ongkeko M, Zeck J, de Brito P. Molecular testing uncovers an Adamantinoma-like Ewing family of tumors in the thyroid: case report and review of literature. AJSP: Rev Rep. 2018;23:8-12.

10. Folpe AL, Goldblum JR, Rubin BP, et al. Morphologic and immunophenotypic diversity in Ewing family tumors: a study of 66 genetically confirmed cases. Am J Surg Pathol. 2005;29:1025-33.

11. Stevens TM, Qarmali M, Morlote D, et al. Malignant Ewing-like neoplasm with an EWSR1-KLF15 fusion: at the crossroads of a myoepithelial carcinoma and a Ewing-like sarcoma. A case report with treatment options. Int J Surg Pathol. 2018. https://doi. org/10.1177/1066896918755009
12. Verma A, Kane S, Vinarkar S, D’Cruz AK. Small cell medullary thyroid carcinoma: a diagnostic dilemma. Indian J Pathol Microbiol. 2017;60:562.

13. Stevens TM, Morlote D, Swensen J, et al. Spindle epithelial tumor with thymus-like differentiation (SETTLE): a next-generation sequencing study. Head Neck Pathol. 2018. https://doi. org/10.1007/s12105-018-0927-1.

14. Bishop JA, Andreasen S, Hang J-F, et al. HPV-related multiphenotypic sinonasal carcinoma. Am J Surg Pathol. 2017;41:1690-701.

15. Wong HH, Wang J. Merkel cell carcinoma. Arch Pathol Lab Med. 2010;134:1711-6.

16. Wenig BM. Squamous cell carcinoma of the upper aerodigestive tract: precursors and problematic variants. Mod Pathol. 2002;15:229.

17. Thompson LD. Olfactory neuroblastoma. Head Neck Pathol. 2009;3:252-9.

18. Haack H, Johnson LA, Fry CJ, et al. Diagnosis of NUT midline carcinoma using a NUT-specific monoclonal antibody. Am J Surg Pathol. 2009;33:984.

19. Lucas DR, Bentley G, Dan ME, et al. Ewing sarcoma vs lymphoblastic lymphoma: a comparative immunohistochemical study. Am J Clin Pathol. 2001;115:11-7.

20. Owosho AA, Estilo CL, Huryn JM, et al. Head and neck round cell sarcomas: a comparative clinicopathologic analysis of 2 molecular subsets: ewing and CIC-rearranged sarcomas. Head Neck Pathol. 2017;11:450-9.

Publisher's Note Springer Nature remains neutral with regard to jurisdictional claims in published maps and institutional affiliations. 